SPIN-2000/30

ITP-UU-00/32

PUTP-1970

hep-th/0011230

\title{
Gravity in Warped Compactifications and the Holographic Stress Tensor
}

\author{
Sebastian de Haro, ${ }^{\star \dagger 1}$ Kostas Skenderis ${ }^{\ddagger 2}$ and Sergey N. Solodukhin ${ }^{\star \star 3}$ \\ * Spinoza Institute, Utrecht University \\ Leuvenlaan 4, 3584 CE Utrecht, The Netherlands \\ $\dagger$ Institute for Theoretical Physics, Utrecht University \\ Princetonplein 5, 3584 CC Utrecht, The Netherlands \\ $¥$ Physics Department, Princeton University \\ Princeton, NJ 08544, USA

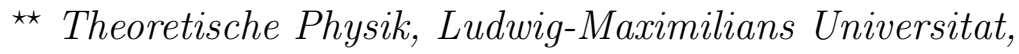 \\ Theresienstrasse 37, D-80333, Munchen, Germany
}

\begin{abstract}
We study gravitational aspects of Brane-World scenarios. We show that the bulk Einstein equations together with the junction condition imply that the induced metric on the brane satisfies the full non-linear Einstein equations with a specific effective stress energy tensor. This result holds for any value of the bulk cosmological constant. The analysis is done by either placing the brane close to infinity or by considering the local geometry near the brane. In the case that the bulk spacetime is asymptotically AdS, we show that the effective stress energy tensor is equal to the sum of the stress energy tensor of matter localized on the brane and of the holographic stress energy tensor appearing in the AdS/CFT duality. In addition, there are specific higher-curvature corrections to Einstein's equations. We analyze in detail the case of asymptotically flat spacetime. We obtain asymptotic solutions of Einstein's equations and show that the effective Newton's constant on the brane depends on the position of the brane.
\end{abstract}

\footnotetext{
${ }^{1}$ e-mail: haro@phys.uu.nl

${ }^{2}$ e-mail: kostas@feynman.princeton.edu

${ }^{3}$ e-mail: soloduk@theorie.physik. uni-muenchen.de
} 


\section{Warped Compactifications and AdS/CFT holography}

In the AdS/CFT correspondence the supergravity partition function is related to the generating functional of conformal field theory (CFT) correlation functions as

$$
Z[\Phi]=\int_{\phi} D \Phi \exp (i S[\Phi])=W_{\text {СFт }}[\phi],
$$

where $\Phi$ denotes collectively all fields and $\phi$ is a field parametrizing the boundary condition of $\Phi$ at infinity. In the conformal field theory the boundary fields $\phi$ are interpreted as sources for CFT operators. In particular, the metric at infinity, $g_{(0)}$, is considered as the source for the stress energy tensor of the dual CFT. The relation (1.1) suffers from divergences and has to be regularized and renormalized.

On the CFT side, there are UV divergences when operators come to coincident points. These correspond to IR divergences on the gravitational side. To regulate the gravitational theory one may cut-off the asymptotically AdS spacetime at some radius $\rho=\epsilon$ near the boundary. One can then compute all infrared divergences. The renormalized theory is obtained by adding counterterms to cancel the infinities and then removing the cut-off.

One may wish, however, to consider situations where the infrared cutoff is kept finite instead of being sent to zero. This is the case in warped compactifications where the AdS spacetime is cut-off by the presence of a brane. In this case, (1.1) does not have any infrared divergences and so one does not need to add counterterms.

In the cut-off spacetime, the induced metric at the boundary $\gamma$ corresponds to a normalizable mode and so one should integrate over it:

$$
\int D \gamma_{\epsilon} \int_{\gamma_{\epsilon}} D G \exp (i S[G])=\int D \gamma_{\epsilon} W_{\mathrm{CFT}}[\gamma, \epsilon]
$$

Under these circumstances, gravity becomes dynamical on the brane, and the brane theory is a CFT coupled to dynamical gravity.

Consider a spacetime $M$ with a boundary $\partial M$. The action in (1.2) is given by ${ }^{4}$

$$
\begin{aligned}
S[\Phi, G]= & \frac{1}{16 \pi G_{d+1}}\left[\int_{M} \mathrm{~d}^{d+1} x \sqrt{G}(R[G]+2 \Lambda)-\int_{\partial M} \mathrm{~d}^{d} x \sqrt{\gamma} 2 K\right] \\
& +\int_{M} \mathrm{~d}^{d+1} x \sqrt{G} \mathcal{L}^{\text {bulk }}+\int_{\partial M} \mathrm{~d}^{d} x \sqrt{\gamma} \mathcal{L}^{\text {bdry }}
\end{aligned}
$$

where $\mathcal{L}^{\text {bulk }}$ denotes the Lagragian for bulk matter and $\mathcal{L}^{\text {bdry }}$ the Lagrangian for matter

\footnotetext{
${ }^{4}$ Our curvature conventions are as follows $R_{i j k}{ }^{l}=\partial_{i} \Gamma_{j k}{ }^{l}+\Gamma_{i p}{ }^{l} \Gamma_{j k}{ }^{p}-i \leftrightarrow j$ and $R_{i j}=R_{i k j}{ }^{k}$. With these conventions the curvature of AdS comes out positive, but we will still use the terminology "space of constant negative curvature". Notice also that we take $\int \mathrm{d}^{d+1} x=\int \mathrm{d}^{d} x \int_{0}^{\infty} \mathrm{d} \rho$ and the boundary is at $\rho=0$. The minus sign in front of the trace of the second fundamental form is correlated with the choice of having $\rho=0$ in the lower end of the radial integration.
} 
living on the boundary. Einstein's equations read ${ }^{5}$ :

$$
\begin{aligned}
R_{\mu \nu}[G]-\frac{1}{2}(R[G]+2 \Lambda) G_{\mu \nu} & =-8 \pi G_{d+1} T_{\mu \nu}^{\text {bulk }}[G] \\
K_{i j}[\gamma]-\gamma_{i j} K[\gamma] & =8 \pi G_{d+1} T_{i j}^{\text {bdry }}[\gamma] .
\end{aligned}
$$

These equations describe the case the bulk spacetime ends on the brane. This is in fact half of the Randall-Sundrum (RS) spacetime [1]. In the RS scenario one glues on the other side of the brane an identical spacetime. Then the substitution

$$
K_{i j} \rightarrow \lim _{\delta \rightarrow 0}\left[K_{i j}(\rho=\epsilon+\delta)-K_{i j}(\rho=\epsilon-\delta)\right],
$$

in (1.5) yields the junction condition (see, for example, [2] for a derivation). $\rho=\epsilon$ is the position of the brane. In the RS context, $K_{i j}(\rho=\epsilon+\delta)=-K_{i j}(\rho=\epsilon-\delta)$ due to the $Z_{2}$ symmetry, so the net effect is to get back (1.5) but with an extra factor of two. In the remainder we will work with equations (1.4) and (1.5) and we will refer to (1.5) as the junction condition.

The usual way [1] of establishing localization of gravity on the brane is to study small fluctuations around a given configuration (such as a flat brane in AdS space) which solves equations (1.4). The equations for small gravitational fluctuations around the solution take the form of a quantum mechanical problem. In terms of the effective quantum mechanical problem the existence of a localized graviton translates into the existence of a normalizable zero-mode solution (this solution is the wave function associated to the graviton localized on the brane). In addition to the zero mode there are additional massive modes. One still has to show that these modes do not drastically change the physics, i.e. that they yield subleading corrections relative to the zero mode. Note that the question of normalizability of the zero mode depends on global properties of the gravitational solution. If the bulk space is asymptotically flat there is still a zero-mode but it is not normalizable. There may still be a quasi-localization due to a collection of low-energy Kaluza-Klein modes [3-5].

The analysis just described is at the linearized level. It is technically involved in this approach to go beyond the linear approximation and demonstrate the full non-linear structure of the gravity localized on the brane. In this paper we use the AdS/CFT duality in order to achieve this goal. Previous works that use the AdS/CFT duality in the RS context include [6-13].

It has been shown in $[14,15]$ that given a metric $g_{(0)}$ on the boundary of AdS one can obtain an asymptotic expansion of the bulk metric near the boundary up to certain order in the radial coordinate (which is regarded as the small parameter in the expansion). The

\footnotetext{
${ }^{5}$ The different signs in the right hand side of these two equations is related to our conventions discussed in the previous footnote.
} 
next order coefficient is left undetemined by the bulk field equations [14]. This coefficient is determined once a symmetric covariantly conserved tensor $T_{i j}^{\mathrm{CFT}}(x)$ with trace equal to the holographic Weyl anomaly is supplied. The tensor $T_{i j}^{\mathrm{CFT}}(x)$ is the holographic stress tensor of the dual conformal field theory [16] (see also [17]). Notice that the CFT stress energy tensor encodes global information too. In particular, regularity of the bulk solution sometimes uniquely fixes $T_{i j}^{\mathrm{CFT}}(x)$.

Let us consider a brane placed close to the AdS boundary. Then one can solve (1.4) by simply considering the asymptotic solution described in the previous paragraph. The junction condition (1.5) then becomes Einstein's equation for the induced metric on the brane. The right-hand side in Einstein's equations is equal to the stress energy tensor due to matter localized on the brane plus the CFT stress energy tensor. In fact, irrespectively of the value of the bulk cosmological constant, Einstein's equations in the bulk plus the junction condition effectively impose Einstein's equations on the brane. This result first appeared in [18]. In particular in all cases the gravitational equations on the brane involve a "holographic stress energy tensor". This can be taken to holographically represent the bulk spacetime.

This paper is organized as follows. In the next section we adopt the results from the AdS/CFT duality to Brane-World scenarios. In particular, we put a brane near the boundary of AdS and obtain the equation that the induced metric on the brane satisfies. In section 3 we place a brane at some (arbitrarily chosen) position in the bulk and analyze the equations near the brane, i.e. we consider the radial distance from the brane as a small parameter. These considerations are valid for any bulk cosmological constant. Finally, in section 4 we consider the case of a brane placed near infinity of an asymptotically flat bulk spacetime.

In this paper we only perform a local analysis. Global issues are important and need to be addressed in order to establish localization of the graviton on the brane. This important issue is left for future study.

\section{Brane gravity from the asymptotic analysis of AdS space}

The asymptotic solutions of the bulk Einstein equation (1.4) in vacuum were worked out in [15] to sufficiently high order. These solutions are best found by writing the bulk metric in the Fefferman-Graham form [14]:

$$
\mathrm{d} s^{2}=\frac{l^{2}}{4 \rho^{2}} \mathrm{~d} \rho^{2}+\frac{l^{2}}{\rho} g_{i j}(\rho, x) \mathrm{d} x^{i} \mathrm{~d} x^{j},
$$


where the metric $g_{i j}$ has the expansion

$$
g(\rho, x)=g_{(0)}+\rho g_{(2)}+\cdots+\rho^{d / 2} g_{(d)}+h_{(d)} \rho^{d / 2} \log \rho+\mathcal{O}\left(\rho^{(d+1) / 2}\right) .
$$

$l^{2}$ is related to the cosmological constant as $\Lambda=-d(d-1) / 2 l^{2}$. Given $g_{(0)}$ all coefficients up to $g_{(d)}$ can be found as local functions of $g_{(0)}$. The coefficient $g_{(d)}$ is undetermined from the gravity equations, and it is related to the stress-energy tensor of the dual CFT:

$$
\left\langle T_{i j}\right\rangle_{\mathrm{CFT}}=\frac{d l^{d-1}}{16 \pi G_{d+1}} g_{(d) i j}+X_{i j}^{(d)}\left[g_{(j)}\right],
$$

where $X_{i j}^{(d)}\left[g_{(j)}\right]$ is a known function of the lower-order coefficients $g_{(j)}, j<d$ [16] (see [19] for a review). The gravitational equations imply that $\left\langle T_{i j}\right\rangle_{\mathrm{CFT}}$ is covariantly conserved and its trace reproduces the conformal anomaly of the boundary CFT.

Let us place a brane close to infinity at constant $\rho=\epsilon$, where $\epsilon$ is small enough for the expansion (2.7) to be a good approximation for the metric in the bulk. Using the results of [16], it is now a simple matter (using (3.4),(3.5),(3.6) and (3.7) of [16]) to see that the junction condition gives Einstein's equation on the brane. For a 3-brane we get:

$$
\begin{aligned}
R_{i j}[\gamma] & -\frac{1}{2} \gamma_{i j}\left(R[\gamma]-\frac{12}{l^{2}}\right)+\frac{1}{4} l^{2} \log \epsilon\left(\frac{1}{12} \nabla_{i} \nabla_{i} R[\gamma]-\frac{1}{4} \nabla^{2} R_{i j}[\gamma]+\frac{1}{24} \gamma_{i j} \nabla^{2} R[\gamma]\right. \\
& \left.+\frac{1}{2} R^{k l}[\gamma] R_{i k j l}[\gamma]-\frac{1}{6} R[\gamma] R_{i j}[\gamma]+\frac{1}{24} \gamma_{i j} R^{2}[\gamma]-\frac{1}{8} \gamma_{i j} R^{k l}[\gamma] R_{k l}[\gamma]\right) \\
& =-16 \pi G_{5} \frac{1}{l}\left(\left\langle T_{i j}[\gamma]\right\rangle_{\mathrm{CFT}}+T_{i j}^{\mathrm{bdry}}[\gamma]\right),
\end{aligned}
$$

where we kept only terms $\mathcal{O}\left(R^{2}\right)$, and there is an explicit dependence on the cutoff through the logarithmic term.

There are several comments in order here:

- In deriving (2.9) it was essential that we added no counterterms to the action. Had we added counterterms, then all the curvature terms in the above formula would have been cancelled. Indeed, these precisely come from the infrared divergent part of the action.

- In the effective Einstein equations the bulk spacetime is represented by the holographic stress energy tensor. In other words, the Brane-World has a purely $d$ dimensional description where the bulk spacetime has been replaced by the cut-off CFT. The CFT couples to matter on the brane only through gravitational interactions.

- The effective Newton's constant is given by

$$
G_{4}=\frac{2 G_{5}}{l}
$$


In the context of the two-sided RS scenario one should divide this result by two (see the discussion after (1.5)).

- The AdS/CFT duality predicts specific $R^{2}$-terms. The terms in (2.9) are derivable from the local action: $\int \mathrm{d}^{d} x a_{(4)}$, where $a_{(4)}$ is the holographic trace anomaly in four dimensions.

- The original expansion in the cutoff becomes an expansion in the brane curvature.

It is straightforward to extend these results to higher dimensions using the results in [16].

In $(2+1)$ dimensions the series in $(2.7)$ terminates at the $\rho^{2}$-term, and one has the exact expression $[20]$

$$
g(x, \rho)=\left(g_{(0)}+\frac{1}{2} g_{(2)} \rho\right)^{2}, \quad g_{(2)}=\frac{1}{2}\left(R g_{(0) i j}+t_{i j}\right),
$$

where $t_{i j}$ is conserved, $\nabla_{(0)}^{j} t_{i j}=0$, and its trace is $\operatorname{Tr} t=-R$. It follows that $t_{i j}$ can be identified as the Liouville stress energy tensor. The holographic stress energy tensor is equal to $\left\langle T_{i j}\right\rangle=\frac{l}{16 \pi G_{3}} t_{i j}$.

Placing an one-brane at $\rho=\epsilon$ and neglecting $\epsilon^{2}$-terms one finds that the junction condition (1.5) implies

$$
\gamma_{i j}=-8 \pi G_{3}\left(T_{i j}^{\text {bdry }}+\left\langle T_{i j}\right\rangle\right),
$$

where $\gamma_{i j}=\frac{1}{\rho} g_{i j}(x, \rho)$ is the induced metric on the brane, and $T_{i j}^{\text {bdry }}$ is the stress tensor of matter on the brane. Note that in two dimensions there is no dynamical theory for just the metric tensor. Gravity induced on the one-brane is of the scalar-tensor type.

In the presence of matter in the bulk, it was shown in [16] that the bulk equations can be solved in the same way. In this case, one again reinterprets the leading in $\epsilon$ terms as giving the terms in the action that determine the dynamics on the brane. For bulk scalar fields of mass $m^{2}=(\Delta-d) \Delta$, the effective brane action is:

$$
\begin{aligned}
S[\gamma, \Phi]= & \int d^{d} x \sqrt{\gamma}\left[\frac{1}{2(2 \Delta-d-2)} \Phi(x, \epsilon) \square_{\gamma} \Phi(x, \epsilon)\right. \\
& \left.+\frac{(d-\Delta)}{2}\left(1+\frac{1}{2(d-1)(2 \Delta-d-2)} R[\gamma]\right) \Phi^{2}(x, \epsilon)\right],
\end{aligned}
$$

where again we only show the first few terms in the low energy expansion. The $d$ dimensional mass receives contributions both from the mass term in $(d+1)$ dimensions but also from the bending of the brane. Notice that a massless field in $d+1$ dimensions remains massless in $d$ dimensions. 


\section{Local analysis}

In the previous section we made use of the asymptotic expansion of the bulk AdS metric (2.7). A similar analysis can be done for a brane located anywhere in the bulk by considering the local geometry near the brane.

Consider the Einstein equations in the bulk

$$
R_{\mu \nu}+\frac{2}{d-1} \Lambda G_{\mu \nu}=0
$$

Near the brane one can use Gaussian normal coordinates. In these coordinates the bulk metric takes the form

$$
\mathrm{d} s^{2}=\mathrm{d} r^{2}+\gamma_{i j}(r, x) \mathrm{d} x^{i} \mathrm{~d} x^{j},
$$

where $r$ stands for the radial coordinate adjusted so that the brane location is at $r=0$. Then the $(i j),(r r)$ and $(r i)$ components of Einstein equations (3.14) read

$$
\begin{gathered}
R_{i j}[\gamma]+\frac{2}{d-1} \Lambda \gamma_{i j}+\frac{1}{2} \partial_{r}^{2} \gamma_{i j}-\frac{1}{2}\left(\partial_{r} \gamma \gamma^{-1} \partial_{r} \gamma\right)_{i j}+\frac{1}{4} \partial_{r} \gamma_{i j} \operatorname{Tr}\left(\gamma^{-1} \partial_{r} \gamma\right)=0 \\
\frac{1}{2} \partial_{r}\left(\operatorname{Tr}\left(\gamma^{-1} \partial_{r} \gamma\right)\right)+\frac{1}{4} \operatorname{Tr}\left(\gamma^{-1} \partial_{r} \gamma\right)^{2}+\frac{2}{d-1} \Lambda=0 \\
\nabla_{j}\left[\gamma^{-1} \partial_{r} \gamma-\operatorname{Tr}\left(\gamma^{-1} \partial_{r} \gamma\right)\right]_{i}^{j}=0
\end{gathered}
$$

Combining the equations (3.16) and (3.17) we find that

$$
R[\gamma]+2 \Lambda+\frac{1}{4}\left(\left[\operatorname{Tr}\left(\gamma^{-1} \partial_{r} \gamma\right)\right]^{2}-\operatorname{Tr}\left(\gamma^{-1} \partial_{r} \gamma\right)^{2}\right)=0
$$

Let $\gamma_{i j}(x, r)$ have the following expansion near the brane:

$$
\gamma=\gamma_{(0)}+\gamma_{(1)} r+\gamma_{(2)} r^{2}+\ldots
$$

Then solving equations (3.16), (3.17) and (3.18) iteratively we find expressions relating the coefficients $\gamma_{(k)}$. From equation (3.16) we find that

$$
\operatorname{Ric}\left[\gamma_{(0)}\right]+\frac{2}{d-1} \Lambda \gamma_{(0)}+\gamma_{(2)}-\frac{1}{2} \gamma_{(1)}^{2}+\frac{1}{4} \gamma_{(1)} \operatorname{Tr} \gamma_{(1)}=0
$$

Equation (3.17) to leading order gives

$$
\operatorname{Tr} \gamma_{(2)}=\frac{1}{4} \operatorname{Tr} \gamma_{(1)}^{2}-\frac{2}{d-1} \Lambda
$$

Taking the trace of (3.20) and using (3.21) one finds

$$
R\left[\gamma_{(0)}\right]+2 \Lambda-\frac{1}{4}\left(\operatorname{Tr} \gamma_{(1)}^{2}-\left(\operatorname{Tr} \gamma_{(1)}\right)^{2}\right)=0 .
$$


This equation can also be obtained from (3.19). Equation (3.17) to the first two orders yields

$$
\begin{gathered}
\nabla^{j} \gamma_{(1) i j}=\nabla_{i} \operatorname{Tr} \gamma_{(1)}, \\
\nabla^{j} \gamma_{(2) i j}=\frac{1}{2} \nabla_{j}\left[\gamma_{(1)}^{2}-\frac{1}{2} \gamma_{(1)} \operatorname{Tr} \gamma_{(1)}-\frac{1}{4} \gamma_{(0)}\left(\operatorname{Tr} \gamma_{(1)}^{2}-\left(\operatorname{Tr} \gamma_{(1)}\right)^{2}\right)\right]_{i}^{j} .
\end{gathered}
$$

Equation (3.23) can be integrated as

$$
\gamma_{(1)}=t_{(1)}+\gamma_{(0)} \operatorname{Tr} \gamma_{(1)},
$$

where $t_{(1) i j}$ is an "integration constant" that satisfies $\nabla^{i} t_{(1) i j}=0$. One can check that (3.24) is automatically satisfied when (3.20) and (3.22) are satisfied.

Forming the Einstein tensor, we obtain

$$
R_{i j}\left[\gamma_{(0)}\right]-\frac{1}{2} \gamma_{(0) i j} R\left[\gamma_{(0)}\right]=\Lambda \gamma_{(0) i j}+T_{i j}
$$

where

$$
T_{i j}=-\frac{2}{d-1} \Lambda \gamma_{(0) i j}-\gamma_{(2) i j}+\frac{1}{2} \gamma_{(1) i j}^{2}-\frac{1}{4} \gamma_{(1) i j} \operatorname{Tr} \gamma_{(1)}-\frac{1}{8} \gamma_{(0) i j}\left[\operatorname{Tr} \gamma_{(1)}^{2}-\left(\operatorname{Tr} \gamma_{(1)}\right)^{2}\right]
$$

Equation (3.24) implies that $T_{i j}$ is covariantly conserved. In addition, equation (3.21) determines the trace of $T_{i j}$,

$$
\operatorname{Tr} T=-2 \Lambda-\frac{(d-2)}{8}\left(\operatorname{Tr} t_{(1)}^{2}-\frac{1}{d-1}\left(\operatorname{Tr} t_{(1)}\right)^{2}\right)
$$

Let us now consider a physical brane with stress tensor $T_{i j}^{\text {bdry }}$ located at $r=0$. Then in addition to equations (3.16), (3.17), (3.18) we have the junction condition (1.5). For the metric (3.15) the second fundamental form is equal to $K_{i j}=\frac{1}{2} \partial_{r} \gamma_{i j}$. From the junction condition (1.5) we get using the equation (3.23)

$$
t_{(1) i j}=16 \pi G_{d+1} T_{i j}^{\text {bdry }} .
$$

The junction condition thus identifies the undetermined covariantly conserved tensor $t_{(1)}$ in (3.25) with the stress tensor of the brane. Notice that conservation of the boundary stress energy tensor is a neccessary condition for this identification.

To summarize we have shown that Einstein's equations in the bulk plus the junction condition lead to Einstein's equations on the brane. The effective stress energy tensor $T_{i j}$ represents both the bulk spacetime and the matter on the brane. Its trace is determined by the matter stress energy tensor on the brane. This is similar to the case discussed in the previous section. There the effective stress energy tensor was a sum of the stress energy tensor of matter localized on the brane of the $\left\langle T_{i j}\right\rangle_{\mathrm{CFT}}$. The latter was taken to 
represent the bulk spacetime, and its trace was fixed to be the holographic conformal anomaly.

The results in this section agree with the results obtained in [18] for $d=4$. To see this, let

$$
\gamma_{(2) i j}=-E_{i j}+\frac{1}{4} \gamma_{(1) i j}^{2}-\frac{2}{d(d-1)} \Lambda \gamma_{(0) i j},
$$

and also let the boundary stress energy tensor be equal to $T_{i j}^{\mathrm{bdry}}=-\lambda \gamma_{i j}^{(0)}+\tau_{i j}$, where $\lambda$ is the tension and $\tau_{i j}$ the matter energy momentum tensor on the brane. Equation (3.30) defines the tensor $E_{i j}$. A short calculation shows that it agrees with the tensor $E_{\mu \nu}$ of [18]. In particular, $E_{i j}$ is traceless and its divergence is equal to $\nabla^{j} E_{i j}=K^{j k}\left(\nabla_{i} K_{j k}-\nabla_{j} K_{i k}\right)$. This agrees with formula (22) of [18]. One can also verify agreement with (17)-(20) of [18].

Note that the above considerations are quite general and valid for any value of the bulk cosmological constant. Note also that when the brane matter consists of only a brane cosmological constant the brane geometry has a constant Ricci scalar.

\section{Asymptotically flat case}

In this section we perform an asymptotic analysis of Einstein's equations with zero cosmological constant similar to the one that has been done for asymptotically AdS spaces in $[14,15]$.

We work in Gaussian normal coordinates. The metric takes the form

$$
\mathrm{d} s^{2}=\mathrm{d} r^{2}+\gamma_{i j}(x, r) \mathrm{d} x^{i} \mathrm{~d} x^{j}
$$

Einstein's equations in this coordinate system are given in equations (3.16), (3.17) and (3.18). We look for an asymptotic solution near infinity. Assuming that the leading part of $\gamma$ near infinity is non-degenerate we find that it scales like $r^{2}$ (to prove this use (3.16)). Restricting ourselves to this case, we look for solutions of the form

$$
\gamma(x, r)=r^{2}\left(g_{(0)}+g_{(2)} \frac{1}{r}+g_{(4)} \frac{1}{r^{2}}+\ldots\right) .
$$

In other words, the bulk metric asymptots to a cone with $g_{(0)}$ the metric on the base. In general, one can include logarithmic terms in (4.32). Such more general asymptotic solutions have been studied in $[21,22]^{6}$. We restrict ourselves to (4.32).

\footnotetext{
${ }^{6}$ In $[21,22]$ the authors look for solutions whose metric coefficients near infinity is given by an expansion in negative powers of the radial coordinate. Coordinate transformations allow one to put the metric in the form $\mathrm{d} s^{2}=N^{2} \mathrm{~d} r^{2}+\gamma_{i j}(x, r) \mathrm{d} x^{i} \mathrm{~d} x^{j}$, with $N=1+\sigma(x) / r$ and $\gamma(x, r)$ as in (4.32). By a further logarithmic transformation one can reach Gaussian normal coordinates but at the expense of introducing logarithmic terms in $\gamma(x, r)$. Our results for $d=3$ agree with the results of $[21,22]$ for $\sigma(x)=0$. We thank Kirill Krasnov for bringing these papers to our attention.
} 
We solve Einstein's equations

$$
R_{\mu \nu}=0,
$$

perturbatively in $1 / r$. The leading order equations imply $[23,24]$ that $g_{(0)}$ should satisfy

$$
R_{(0) i j}+(d-1) g_{(0) i j}=0
$$

This means that the space at infinity is described by an Einstein metric of constant positive scalar curvature. In particular, for Euclidean signature the standard metric on the unit sphere $S^{d-1}$ satisfies this equation. Then the leading part of the bulk metric (4.31), (4.32) is just Euclidean $R^{d}$ space. In the Lorentzian signature equation (4.34) is solved by the de Sitter space. Thus, already at leading order, we find an important difference between the cases of asymptotically flat spacetime and of asymptotically AdS spacetimes. Whereas in the latter case one could choose the boundary metric arbitrarily, in the former case the boundary metric has to satisfy (4.34).

To next order we find

$$
\begin{aligned}
& \nabla^{j} g_{(2) i j}=\nabla_{i} \operatorname{Tr} g_{(2)}, \\
& d g_{(2)}+2 \operatorname{Ric}_{(2)}-g_{(0)} \operatorname{Tr} g_{(2)}=0,
\end{aligned}
$$

where

$$
\operatorname{Ric}[\gamma]=\operatorname{Ric}_{(0)}+\frac{1}{r} \operatorname{Ric}_{(2)}+\frac{1}{r^{2}} \operatorname{Ric}_{(4)} \cdots
$$

and

$$
R_{(2) i j}=-\frac{1}{2}\left[\nabla_{i} \nabla_{j} \operatorname{Tr} g_{(2)}-\nabla^{2} g_{(2) i j}+2(d-1) g_{(2) i j}+2 R_{(0) i k j l} g_{(2)}^{k l}\right],
$$

where indices raised and lowered by $g_{(0)}$. In deriving this equation, (4.34) and (4.35) were used. Then equation (4.36) becomes

$$
\nabla_{i} \nabla_{j} \operatorname{Tr} g_{(2)}-\nabla^{2} g_{(2) i j}+(d-2) g_{(2) i j}+g_{(0) i j} \operatorname{Tr} g_{(2)}+2 R_{(0) i k j l} g_{(2)}^{k l}=0 .
$$

Notice that this equation leaves undetermined the trace of $g_{(2)}$. Let us define

$$
t_{i j}=g_{(2) i j}-g_{(0) i j} \operatorname{Tr} g_{(2)} .
$$

It follows from the (4.35) that $\nabla^{i} t_{i j}=0$.

To the next order we find the equations

$$
\begin{aligned}
g_{(4)} & =-\frac{1}{2} \operatorname{Ric}_{(4)}+\frac{1}{2} g_{(0)} \operatorname{Tr} g_{(4)}-\frac{1}{4} g_{(0)} \operatorname{Tr} g_{(2)}^{2}+\frac{1}{4} g_{(2)}^{2}+\frac{1}{8} g_{(2)} \operatorname{Tr} g_{(2)}, \\
\operatorname{Tr} g_{(4)} & =\frac{1}{4} \operatorname{Tr} g_{(2)}^{2}, \\
\nabla^{j} g_{(4) i j} & =\frac{1}{2} \nabla_{j}\left[g_{(2)}^{2}-\frac{1}{2} g_{(2)} \operatorname{Tr} g_{(2)}-\frac{1}{4} g_{(0)}\left(\operatorname{Tr} g_{(2)}^{2}-\left(\operatorname{Tr} g_{(2)}\right)^{2}\right)\right]_{i}^{j},
\end{aligned}
$$


and

$$
\begin{aligned}
R_{(4) i j}= & \frac{1}{2}\left[-\frac{1}{4} \nabla_{i} \nabla_{j} \operatorname{Tr} g_{(2)}^{2}-\nabla^{k} \nabla_{i} g_{(4) j k}-\nabla^{k} \nabla_{j} g_{(4) i k}+\nabla^{2} g_{(4) i j}\right. \\
& +g_{(2)}^{k l}\left[\nabla_{l} \nabla_{i} g_{(2) j k}+\nabla_{l} \nabla_{j} g_{(2) i k}-\nabla_{l} \nabla_{k} g_{(2) i j}\right] \\
& +\frac{1}{2} \nabla^{k} \operatorname{Tr} g_{(2)}\left(\nabla_{i} g_{(2) j k}+\nabla_{j} g_{(2) i k}-\nabla_{k} g_{(2) i j}\right) \\
& \left.+\frac{1}{2} \nabla_{i} g_{(2) k l} \nabla_{j} g_{(2)}^{k l}+\nabla_{k} g_{(2) i l} \nabla^{l} g_{(2) j}{ }^{k}-\nabla_{k} g_{(2) i l} \nabla^{k} g_{(2) j}{ }^{l}\right] .
\end{aligned}
$$

It may seem that by taking the trace of (4.41) and using (4.42) and (4.41) one obtains a new equation for $g_{(2)}$. However, it turns out that the resulting equation is automatically satisfied. The same is true when taking the trace of (4.36) and using (4.38).

The equations we obtained look similar to the equations one gets in the case of asymptotically AdS spacetimes. There are important differences, however. In the case of asymptotically AdS spacetimes the equations were algebraic, and they could be solved up to order $\rho^{d}$. The coefficient $g_{(d)}$ was undetermined except for its trace and divergence. In the case at hand the equations for the coefficients are differential, and it is the trace of $g_{(2)}$ which is undetermined.

Let us place the brane at a fixed large radius $r=r_{0}>>1$. Then expanding the Einstein tensor for the induced metric $\gamma_{i j}$ we find that

$$
R_{i j}[\gamma]-\frac{1}{2} \gamma_{i j} R[\gamma]=(d-2)\left(\frac{d-1}{2 r_{0}^{2}} \gamma_{i j}+\frac{1}{2 r_{0}} t_{i j}\right)+\mathcal{O}\left(1 / r_{0}^{2}\right)
$$

where $t_{i j}$ is given in (4.40). On the other hand we have

$$
K_{i j}-\gamma_{i j} K=-\frac{d-1}{r_{0}} \gamma_{i j}-\frac{1}{2} t_{i j}+\mathcal{O}\left(1 / r_{0}^{2}\right)
$$

Notice that this is the Brown-York stress energy tensor [25]. Thus, up to the leading divergence in $r_{0} \rightarrow \infty, t_{i j}$ is equal to the Brown-York stress energy tensor. The junction condition on the brane gives a relation between $t_{i j}$ and the stress tensor $T_{i j}^{\mathrm{bdry}}$ of matter fields on the brane. Plugging back to (4.45) we find

$$
R_{i j}[\gamma]-\frac{1}{2} \gamma_{i j} R[\gamma]=-\frac{(d-2)(d-1)}{2 r_{0}^{2}} \gamma_{i j}-\frac{(d-2) 8 \pi G_{d+1}}{r_{0}} T_{i j}^{\mathrm{bdry}}+\mathcal{O}\left(1 / r_{0}^{2}\right),
$$

i.e. we get Einstein's equations with negative cosmological constant $\Lambda=-\frac{(d-1)(d-2)}{2 r_{0}^{2}}$ and Newton's constant $G_{d}=\frac{(d-2) G_{d+1}}{r_{0}}$. The position of the brane becomes the AdS radius of gravity on the brane. Notice also that the formula for $G_{d}$ is the same with formula (2.10) with $l$ replaced by $r_{0}$.

Acknowledgements: KS is supported in part by the NSF grant PHY-9802484. SS is supported by the DFG Priority Programme SPP-1096. SdH would like to thank Soo- 
Jong Rey for hospitality and interesting discussions during his stay at Seoul National University.

\section{References}

[1] L. Randall and R. Sundrum, "An Alternative to Compactification", Phys.Rev.Lett. 83 (1999) 4690-4693, hep-th/9906064.

[2] S.K. Blau, E.I Guendelman and A.H. Guth, "Dynamics of False-vacuum bubbles", Phys. Rev D35 (1987) 1747.

[3] R. Gregory, V. A. Rubakov, and S. M. Sibiryakov, "Opening Up Extra Dimensions at Ultra-Large Scales," Phys. Rev. Lett. 84 (2000) 5928; hep-th/0002072.

[4] C. Csaki, J. Erlich and T.J. Hollowood, "Quasilocalization of gravity by resonant modes", Phys.Rev.Lett. 84 (2000) 5932-5935, hep-th/0002161.

[5] G. Dvali, G. Gabadadze and M. Porrati, "Metastable gravitons and infinite volume extra dimensions", Phys.Lett. B484 (2000), 112-118, hep-th/0002190.

[6] H. Verlinde, "Holography and Compactification", hep-th/9906182.

[7] S.S. Gubser, "AdS/CFT and gravity", hep-th/9912001.

[8] S. B. Giddings, E. Katz, and L. Randall, "Linearized Gravity in Brane Backgrounds," JHEP 0003 (2000) 023; hep-th/0002091.

[9] S.W. Hawking, T. Hertog and H.S. Reall, "Brane New World", Phys.Rev. D62 (2000) 043501, hep-th/0003052.

[10] M.J. Duff and J.T. Liu, "Complementarity of the Maldacena and Randall-Sundrum Pictures", Phys. Rev. Lett. 85 (2000) 2052, hep-th/0003237.

[11] L. Anchordoqui, C. Nunez and K. Olsen, "Quantum Cosmology and AdS/CFT", JHEP 0010 (2000) 050, hep-th/0007064.

[12] S.B. Giddings and E. Katz, "Effective theories and black hole production in warped compactifications", hep-th/0009176.

[13] N.S. Deger and A. Kaya, "AdS/CFT and Randall-Sundrum Model Without a Brane", hep-th/0010141.

[14] C. Fefferman and C. Robin Graham, 'Conformal Invariants', in Elie Cartan et les Mathématiques d'aujourd'hui (Astérisque, 1985) 95. 
[15] M. Henningson and K. Skenderis, "The holographic Weyl anomaly", JHEP 9807 (1998) 023, hep-th/9806087; "Holography and the Weyl Anomaly", hep-th/9812032.

[16] S. de Haro, K. Skenderis and S.N. Solodukhin, "Holographic Reconstruction of Spacetime and Renormalization in the AdS/CFT Correspondence", to appear in Commun. Math. Phys., hep-th/0002230.

[17] K. Bautier, F. Englert, M. Rooman and Ph. Spindel, "The Fefferman-Graham Ambiguity and AdS Black Holes", Phys. Lett. B479 (2000) 291-298, hep-th/0002156.

[18] T. Shiromizu, K. Maeda and M. Sasaki, "The Einstein Equations on the 3-Brane World", Phys.Rev. D62 (2000) 024012, gr-qc/9910076.

[19] K. Skenderis, "Asymptotically Anti-de Sitter spacetimes and their stress energy tensor", hep-th/0010138.

[20] K. Skenderis and S.N. Solodukhin, "Quantum effective action from the AdS/CFT correspondence", Phys. Lett. B432 (2000) 316-322, hep-th/9910023.

[21] R. Beig and B.G. Schmidt, "Einstein's equations near Spatial Infinity", Commun.Math.Phys. 87 (1982) 65-80.

[22] R. Beig, "Integration of Einstein's equations near spatial infinity", Proc.R.Soc.Lond. bf A 391 (1984) 295-304.

[23] G.W. Gibbons, D.N. Page and C.N. Pope, "Einstein metrics on $S^{3}, R^{3}$ and $R^{4}$ buddles", Commun.Math.Phys. 127 (1990) 529.

[24] S.N. Solodukhin, "How to make the gravitational action on non-compact space finite", Phys.Rev. D62 (2000), 044016, hep-th/9909197.

[25] J.D. Brown and J.W. York, "Quasilocal energy and conserved charges derived from the gravitational action", Phys.Rev. D47 (1993), 1407-1419. 